\title{
Cevipabulin Fumarate
}

National Cancer Institute

\section{Source}

National Cancer Institute. Cevipabulin Fumarate. NCI Thesaurus. Code C73815.

The fumarate salt of cevipabulin, a synthetic, water soluble tubulin-binding agent with potential antineoplastic activity. Cevipabulin appears to bind at the vinca-binding site on tubulin, but seems to act more similar to taxane-site binding agents in that it enhances tubulin polymerization and does not induce tubulin depolymerization. The disruption in microtubule dynamics may eventually inhibit cell division and reduce cellular growth., a small, water soluble, synthetic tubulin-binding agent with potential antineoplastic activity. Cevipabulin appears to bind at the vinca-binding site on tubulin, but seems to act more similar to taxane-site binding agents in that it enhances tubulin polymerization and does not induce tubulin depolymerization. The disruption in microtubule dynamics may eventually inhibit cell division and may reduce cellular growth. 